\title{
Recombinant human thrombomodulin inhibits neutrophil extracellular trap formation in vitro
}

\author{
Yasuyo Shimomura1", Mika Suga', Naohide Kuriyama ${ }^{1,3}$, Tomoyuki Nakamura $^{1}$, Toshikazu Sakai ${ }^{1}$, Yu Kato ${ }^{1}$, \\ Yoshitaka Hara', Chizuru Yamashita', Hiroshi Nagasaki ${ }^{2}$, Masao Kaneki ${ }^{3}$ and Osamu Nishida ${ }^{1}$
}

\begin{abstract}
The aim of this study was to investigate the effects of recombinant human-soluble thrombomodulin (rTM) on lipopolysaccharide (LPS)-induced, platelet-dependent neutrophil extracellular trap (NET) formation (NETosis). Human peripheral blood neutrophils and platelets were co-incubated with or without LPS $(0.2 \mu \mathrm{g} / \mathrm{ml})$ in the presence and absence of rTM $(2 \mu \mathrm{g} / \mathrm{ml})$. NETosis was confirmed by immunostaining and confocal microscopy. In the absence of platelets, LPS did not induce NETosis in the neutrophils. NETosis, however, was induced by LPS when neutrophils were co-cultured with platelets (64 \% of neutrophils). Notably, rTM was able to fully inhibit NETosis in neutrophils cultured with platelets and in the presence of LPS. rTM did not induce NETosis in this co-culture system $(p<0.01$ versus LPS in the absence of rTM). These results show that rTM can suppress LPS-induced platelet-dependent NETosis in vitro.
\end{abstract}

Keywords: Disseminated intravascular coagulation, Innate immunity, Neutrophil extracellular traps, Sepsis, Thrombomodulin

\section{Introduction}

Neutrophil extracellular traps (NETs) are important for local defense against invading pathogens [1]. However, NET formation, also known as NETosis, can cause or exacerbate hypercoagulability, microvascular thrombosis, and vascular endothelial dysfunction in critical illness, including sepsis [2].

Thrombomodulin (TM) is mainly expressed on the cell surface of endothelial cells, but the soluble form of TM also exists in the circulation [3]. TM exerts protective effects against endothelial dysfunction and damage associated with sepsis and coagulopathy [4]. However, the mechanisms underlying the protective effects of TM in endothelial cells are not completely understood. Recently, recombinant human-soluble TM (rTM) has been used for the treatment of patients with sepsis-associated disseminated intravascular coagulation (DIC) in

\footnotetext{
* Correspondence: yasuyo@fujita-hu.ac.jp

${ }^{1}$ Department of Anesthesiology and Critical Care Medicine, Fujita Health

University School of Medicine, 1-98 Dengakugakubo, Kutsukake-cho,

Toyoake, Aichi 470-1192, Japan

Full list of author information is available at the end of the article
}

Japan, and rTM treatment has shown protective effects [5].

A previous study demonstrated that lipopolysaccharide (LPS) induces NET formation in a platelet-dependent manner [2]. Consistent with this previous study, we found that while neither LPS nor platelets alone caused NETosis in cultured neutrophils, LPS co-cultured with neutrophils and platelets markedly induced NETosis. These results clearly indicate that LPS-induced NET formation is platelet-dependent.

We noticed that NET formation induced by both LPS and activated platelets resembled a serious condition of inflammation in response to infection, such as during septic shock and sepsis-associated DIC. However, the effects of rTM on NET formation have not been studied. Therefore, in this study, we investigated the effects of rTM on NETosis in the presence of both LPS and platelets in vitro.

(c) 2016 The Author(s). Open Access This article is distributed under the terms of the Creative Commons Attribution 4.0 International License (http://creativecommons.org/licenses/by/4.0/), which permits unrestricted use, distribution, and reproduction in any medium, provided you give appropriate credit to the original author(s) and the source, provide a link to the Creative Commons license, and indicate if changes were made. The Creative Commons Public Domain Dedication waiver (http://creativecommons.org/publicdomain/zero/1.0/) applies to the data made available in this article, unless otherwise stated. 


\section{Methods}

Isolation of human neutrophils and platelets

Peripheral blood was collected in EDTA-containing Vacutainers from ten healthy volunteers. Neutrophils were isolated using Polymorphprep (Axis-Shield, Dundee, UK). For platelet isolation, $2 \mathrm{ml}$ of whole blood containing $3.2 \%$ sodium citrate solution was centrifuged at $300 \mathrm{~g}$ for $5 \mathrm{~min}$ at $4{ }^{\circ} \mathrm{C}$. Platelet-rich plasma was centrifuged at $1000 \mathrm{~g}$ for $15 \mathrm{~min}$ at $4{ }^{\circ} \mathrm{C}$, the supernatants were removed, and the cell pellets were resuspended in PBS.

\section{In vitro NET formation}

In vitro NETosis was induced as previously described [6] with minor modifications. In brief, neutrophils $\left(5 \times 10^{4}\right)$ were incubated in PBS containing $10 \%$ fetal bovine serum with or without platelets $\left(5 \times 10^{5}\right)$. LPS (Escherichia coli 0111, 125-05201, Wako Pure Chemical Industries, Ltd., Osaka, Japan) was added at $0.2 \mu \mathrm{g} / \mathrm{ml}$ to stimulate cells. To investigate the effect of rTM on the inhibition of NETosis, incubated cells were treated with rTM (2, 10, or $50 \mu \mathrm{g} / \mathrm{ml}(39,192$, or $960 \mathrm{nM})$, ART-123,

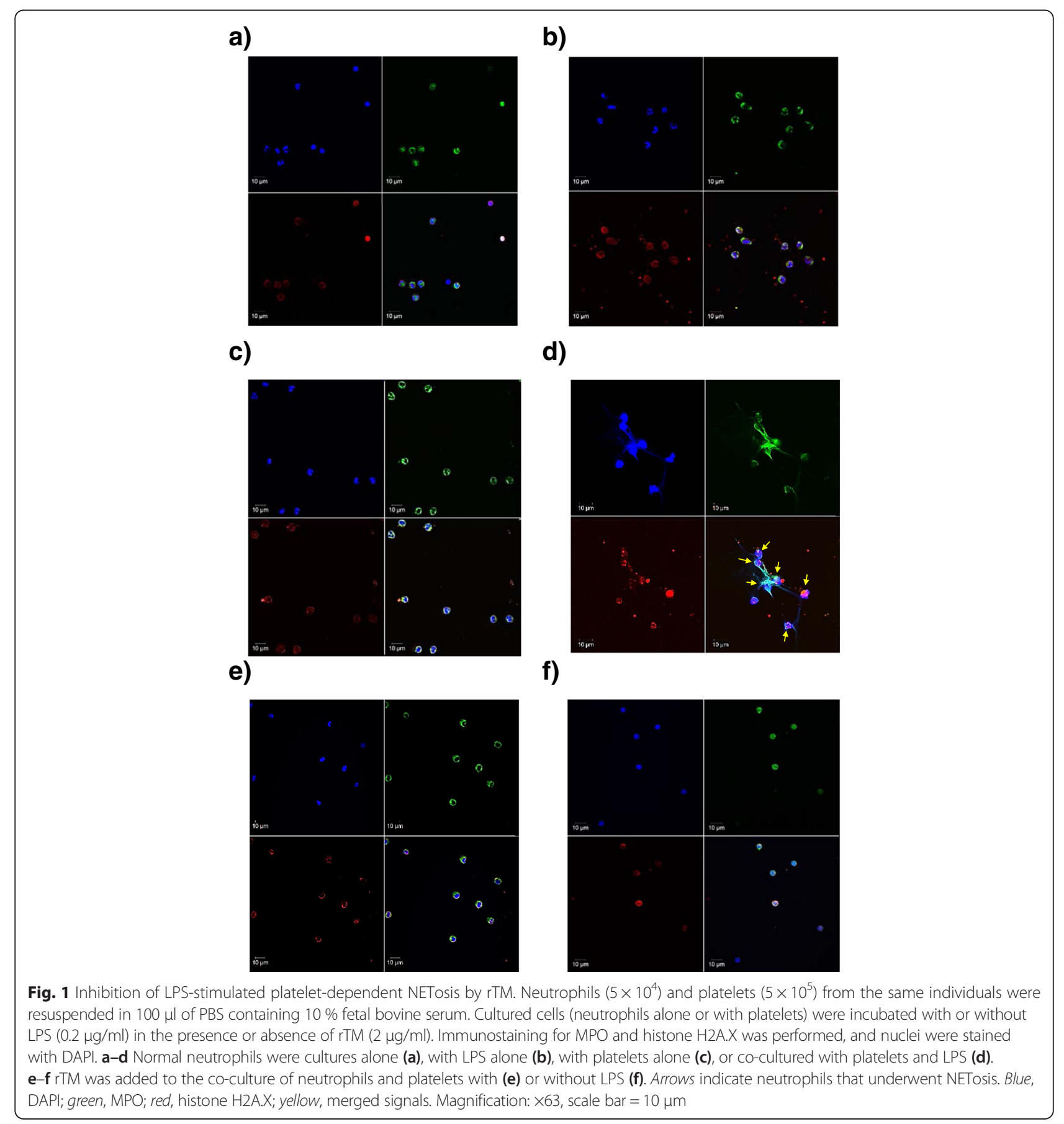


provided by Asahi Kasei Pharma Corp., Tokyo, Japan) immediately after LPS stimulation and incubated for $30 \mathrm{~min}$ at $37{ }^{\circ} \mathrm{C}$ in $5 \% \mathrm{CO}_{2}$. rTM concentrations were chosen based on previous studies [7, 8]. Clinically, rTM $(0.06 \mathrm{mg} / \mathrm{kg})$ was administered intravenously once daily for six consecutive days, and Cmax (maximum plasma drug concentration) reached $1600 \mathrm{ng} / \mathrm{ml}$ [7]. Furthermore, previous studies used rTM concentrations of 2 or $20 \mu \mathrm{g} / \mathrm{ml}$ in vitro [8]. Therefore, rTM was added at the concentrations of 2,10 , or $50 \mu \mathrm{g} / \mathrm{ml}$ in this study.

\section{Immunostaining and confocal microscopy}

Cells were stained with an anti-myeloperoxidase (MPO) (ab45977, Abcam, Cambridge, MA, USA) or histone H2A.X antibody (sc-54607, Santa Cruz Biotechnology, Santa Cruz, CA, USA), followed by species-specific secondary antibodies conjugated with Alexa Fluor Dyes (Invitrogen, Life Technologies Japan, Tokyo, Japan). DNA was stained with DAPI (D1306, Invitrogen, Life Technologies). NETosis was evaluated by the release of MPO, histone H2A.X, and DNA from the neutrophils using a confocal microscope (LSM 710 confocal microscope; Carl Zeiss MicroImaging, Jena, Germany). The numbers of NETs and total neutrophils were counted, and the percentage of NETs in the total neutrophils was calculated as previously described $[9,10]$.

\section{Statistical analyses}

The percentage of NETs was expressed as median with interquartile range (IQR) and compared using the Kruskal-Wallis one-way analysis of variance on ranks.

\section{Results}

The number of neutrophils was counted in the observation view field of a microscope image.

LPS did not induce NETosis when neutrophils were not co-cultured with platelets. Without LPS stimulation, NETosis did not occur in the co-culture of neutrophils and platelets (Fig. 1a-c). However, when neutrophils cultured in the presence of platelets were stimulated by LPS, neutrophils released chromatin (observed by histone H2A.X staining) lined with granular components (observed by MPO staining), creating fibrous nets in the cytoplasm. These observations confirmed that neutrophils emitted NETs (Fig. 1d). This confirms previous observations showing that the NETosis induced by LSP is platelet-dependent [2].

To investigate the effect of rTM on LPS-induced platelet-dependent NETosis, rTM was added to the coculture of neutrophils and platelets. In the presence of rTM $(2,10$, or $50 \mu \mathrm{g} / \mathrm{ml})$, LPS failed to induce NETosis. Our results showed that $\mathrm{rTM}$ at $2 \mu \mathrm{g} / \mathrm{ml}$ was sufficient to block LPS-induced NETosis (Fig. 1e). The results showed that rTM inhibited the LPS-induced platelet- dependent NETosis, as indicated by the unchanged intracellular localization of DNA, MPO, and H2A.X (Fig. 1e). On the other hand, rTM did not induce NETosis in the absence of LPS (Fig. 1f).

Quantification of NETosis in the co-culture of neutrophils and platelets revealed that the addition of LPS to the neutrophil-platelet co-culture induced NETosis in $64 \%$ of neutrophils (IQR, 36-82 \%) (Fig. 2). However, rTM at $2 \mu \mathrm{g} / \mathrm{ml}$ was sufficient to block LPS-induced NETosis to $0 \%$ (IQR, $0.0-0.03 \%])(p<0.01$ versus LPS alone) (Fig. 2). Together, our findings demonstrate that rTM is capable of suppressing NETosis in vitro.

\section{Discussion}

Our results showed that LPS stimulation did not induce NETosis from neutrophils without co-culture with platelets. These results are consistent with findings from a previous study [2]. Importantly, our study revealed that LPS-induced platelet-dependent NETosis was inhibited by rTM.

NETosis in the microvasculature is an important molecular event causing endothelial dysfunction [2]. Our data raise the possibility that endogenous TM both in

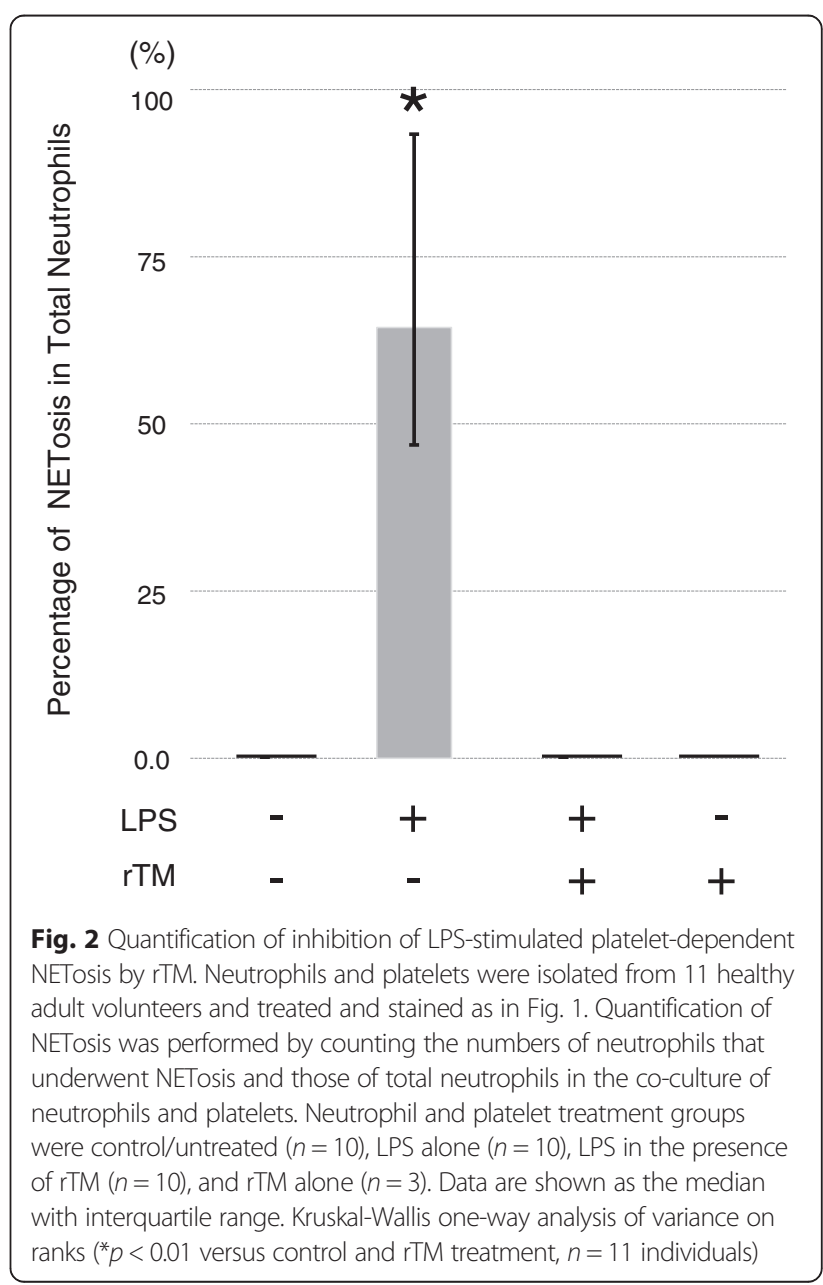


the endothelial cells and in the circulation (the soluble form of TM) may decrease NETosis and thereby prevent endothelial dysfunction contributing to the improvements in the clinical outcome of patients with sepsisassociated DIC.

Yipp et al. described two types of NETosis that occur via two distinct pathways [11]. Phorbol 12-myristate 13acetate-induced NETosis is associated with nicotinamide adenine dinucleotide phosphate oxidase-dependent cellular death. This pathway requires hours for release of NETs. In contrast, microbial exposure or LPS-induced NETosis occurs rapidly $(<30 \mathrm{~min})[2,12]$. Moreover, the direct interaction of activated platelets with neutrophils causes NETosis, particularly when platelets are activated via Toll-like receptor (TLR) 4-mediated signaling [2]. Coagulopathy is an integral component of the development of severe sepsis. To investigate the effects of rTM on NETosis, we therefore used the LPS-stimulated platelet-dependent NETosis, a clinically relevant model of NETs in the context of coagulopathy and sepsis.

As a limitation of these in vitro experiments, however, the mechanisms of the rTM-mediated inhibition of NETosis are not clear and remain to be determined. It is possible that rTM may exert an inhibitory effect against TLR4-mediated signaling. We are currently attempting to clarify the mechanisms by which rTM inhibits LPSinduced NETosis.

Our findings suggest that rTM and possibly endogenous TM may have a novel role in the regulation of NETosis. These data raise the possibility that the inhibition of NETosis may contribute to the protective effects of rTM in patients with sepsis and/or DIC. Further studies are required to determine whether rTM inhibits NETosis in vivo in patients with sepsis and/or DIC.

\section{Abbreviations}

Cmax, maximum plasma drug concentration; DIC, disseminated intravascular coagulation; IQR, interquartile range; LPS, lipopolysaccharide; MPO, myeloperoxidase; NETs, neutrophil extracellular traps; rTM, recombinant human-soluble thrombomodulin; TLR, Toll-like receptor

\section{Acknowledgements}

This work was supported in part by the Grants-in-Aid for Scientific Research (C), JSPS, Japan (Grant \# 25462418), and a research grant (2), Fujita Academy, Japan.

\section{Funding}

The authors were supported by JSPS KAKENHI for data collections and analysis.

\section{Availability of data and materials}

The datasets supporting the conclusions of this article are included within the article.

\section{Authors' contributions}

YS designed, performed all of the experiments, and wrote the manuscript. MS, NK, TN, TS, YK, and YH performed the research. CY and $\mathrm{HN}$ assisted with the experiments. ON conceived the project and secured funding, and ON and MK participated in the study design and manuscript writing. All authors read and approved the final manuscript.

\section{Authors' information}

YS is a senior assistant professor. MS, TN, and $\mathrm{YH}$ are assistant professors, and $\mathrm{ON}$ is a professor and chairman of the Department of Anesthesiology and Critical Care Medicine, Fujita Health University School of Medicine. NK is an assistant professor in the Department of Anesthesiology and Critical Care Medicine, Fujita Health University School of Medicine, and a research fellow in the Department of Anesthesia, Critical Care and Pain Medicine, Massachusetts General Hospital, Harvard Medical School. TS is a student at the Fujita Health University Graduate School of Medicine. YK is a student at the Fujita Health University Graduate School of Health Sciences. CY is an associate professor at the Fujita Health University Hospital. HN is a professor at the Department of Physiology I, Fujita Health University School of Medicine. MK is an associate professor at the Department of Anesthesia, Critical Care and Pain Medicine, Massachusetts General Hospital, Harvard Medical School.

\section{Competing interests}

YS received an honorarium for a lecture from Asahi Kasei Pharma Corporation (Tokyo, Japan). The other authors have no conflict of interest. Asahi Kasei Pharma Corporation provided recombinant human-soluble thrombomodulin (ART-123). The investigation was investigator-initiated, and the provider was not involved in the study design or conduct and had no role in manuscript preparation.

\section{Consent for publication}

Not applicable.

\section{Ethics approval and consent to participate}

The study was approved by the Institutional Review Board of Fujita Health University (\#09-157). Neutrophils and platelets were isolated from the peripheral blood of healthy volunteer donors who gave informed consent.

\section{Author details}

'Department of Anesthesiology and Critical Care Medicine, Fujita Health University School of Medicine, 1-98 Dengakugakubo, Kutsukake-cho, Toyoake, Aichi 470-1192, Japan. ${ }^{2}$ Department of Physiology I, Fujita Health University School of Medicine, 1-98 Dengakugakubo, Kutsukake-cho, Toyoake 470-1192, Japan. ${ }^{3}$ Department of Anesthesia, Critical Care and Pain Medicine, Massachusetts General Hospital, Harvard Medical School, 149 13th Street, Charlestown, MA 02129, USA.

Received: 17 December 2015 Accepted: 18 July 2016

Published online: 22 July 2016

\section{References}

1. Brinkmann V, Reichard U, Goosmann C, Fauler B, Uhlemann Y, Weiss DS, et al. Neutrophil extracellular traps kill bacteria. Science. 2004;303:1532-5.

2. Clark SR, Ma AC, Tavener SA, MCDonald B, Goodarzi Z, Kelly MM, et al. Platelet TLR4 activates neutrophil extracellular traps to ensnare bacteria in septic blood. Nat Med. 2007;13:463-9.

3. Ohlin AK, Larsson K, Hansson M. Soluble thrombomodulin activity and soluble thrombomodulin antigen in plasma. J Thromb Haemost. 2005:3:976-92.

4. Schouten M, Wiersinga WJ, Levi M, van der Poll T. Inflammation, endothelium, and coagulation in sepsis. J Leukoc Biol. 2008;83:536-45.

5. Yamakawa K, Ogura H, Fujimi S, Morikawa M, Ogawa Y, Mohri T, et al. Recombinant human soluble thrombomodulin in sepsis-induced disseminated intravascular coagulation: a multicenter propensity score analysis. Intensive Care Med. 2013;39:644-52.

6. Caudrillier A, Kessenbrock K, Gilliss BM, Nguyen JX, Marques MB, Monestier $M$, et al. Platelets induce neutrophil extracellular traps in transfusion-related acute lung injury. J Clin Invest. 2012;122:2661-71.

7. Tsuruta K, Yamada Y, Serada M, Tanigawara Y. Model-based analysis of covariate effects on population pharmacokinetics of thrombomodulin alfa in patients with disseminated intravascular coagulation and normal subjects. J Clin Pharmacol. 2011;51:1276-85.

8. Saigo K, Mori C, Iwamoto S, Shirai K, Uematsu T, Takenokuchi M, et al. Recombinant thrombomodulin does not impair neutrophil functions. J Thromb Thrombolysis. 2015;39:536-8.

9. Buchanan JT, Simpson AJ, Aziz RK, Liu GY, Kristian SA, Kotb M, et al. DNase expression allows the pathogen group A Streptococcus to escape killing in neutrophil extracellular traps. Curr Biol. 2006;16:396-400. 
10. Etulain J, Martinod K, Wong SL, Cifuni SM, Schattner M, Wagner DD.

P-selectin promotes neutrophil extracellular trap formation in mice. Blood. 2015;126:242-6.

11. Yipp BG, Kubes P. NETosis: how vital is it? Blood. 2013;122:2784-94.

12. Pilsczek FH, Salina D, Poon KK, Fahey C, Yipp BG, Sibley CD, et al. A novel mechanism of rapid nuclear neutrophil extracellular trap formation in response to Staphylococcus aureus. J Immunol. 2010;185:7413-25.

Submit your next manuscript to BioMed Central and we will help you at every step:

- We accept pre-submission inquiries

- Our selector tool helps you to find the most relevant journal

- We provide round the clock customer support

- Convenient online submission

- Thorough peer review

- Inclusion in PubMed and all major indexing services

- Maximum visibility for your research

Submit your manuscript at www.biomedcentral.com/submit

C) Biomed Central 